presentation. On the night prior to presentation, swelling of the arm was noted with associated pain, which was worse on movement. Paraesthesia of the fingers of the affected limb was also noted.

Past history Her past history was notable for asthma (mild, no previous admissions) and hypermobility syndrome.

Medications There was no known drug allergies. She was not on any regular medications, including any form of contraceptive.

Vaccinations were up to date. Family history was notable for Raynaud's disease. There was no history of venous thromboembolism or any coagulation disorders.

Examination On examination, a visibly swollen right upper limb was noted, with a $3 \mathrm{~cm}$ difference in circumference and bruising as described. Dilated tortuous veins were visible. The patient was tender to palpation over the biceps muscle and site of tendon insertion. Full power and range of motion was noted at both shoulder and elbow joints. Radial pulses were present and equal. The remaining systemic examination was unremarkable.

Investigations Bloods normal, including coagulation

US Doppler upper limb no obvious DVT. However, dilatation of basilic vein proximally, with a possible varicosity was noted

MRA right upper limb large thrombus of right subclavian vein, protruding into right brachiocephalic vein and axillary vein

CT pulmonary angiogram: significant reduction in costoclavicular distance (right $>$ left), suggestive of a thoracic outlet obstruction

Treatment Therapeutic subcutaneous low molecular weight heparin was commenced. The patient was admitted for further management, which included thrombectomy and heparin infusion.

Discussion Paget-Schroetter syndrome, or primary 'spontaneous' DVT of the upper limb, is a condition which typically affects otherwise healthy individuals. It occurs as a result of anatomical abnormalities of the thoracic outlet, which results in compression of the axillosubclavian venous system and subsequent thrombosis. It is a rare condition that is, however, important to recognize promptly as early detection and treatment have been found to reduce long-term sequelae.

This case, while rare, highlights the importance to us, as paediatric physicians, to consider in differential diagnosess, disorders and problems that would typically be more attributable to adult populations.

\section{GP56 SEVERE HYPERCALCEMIC CRISIS IN AN INFANT WITH IDIOPATHIC INFANTILE HYPERCALCEMIA CAUSED BY MUTATION IN CYP24A1 GENE}

'Orsolya-Adrienn Rácz* ${ }^{2}$ Francesco Emma, ${ }^{3}$ Martin Konrad, ${ }^{4}$ Otilia Fufezan, ${ }^{1}$ Bogdan Bulată, ${ }^{1}$ Adriana Bungardi, ${ }^{1}$ Simona Căinap. ${ }^{1} 2 n d$ Pediatric Clinic, University of Medicine and Pharmacy Iuliu Hatieganu, Cluj-Napoca, Romania; ${ }^{2}$ Division of Nephrology, Bambino Gesu Children's Hospital, IRCCS, Rome, Italy; ${ }^{3}$ Department of Pediatrics, University of Münster, Münster, Germany; ${ }^{4}$ Department of Radiology, University of Medicine and Pharmacy Iuliu Hatieganu, Cluj-Napoca, Romania

\subsection{6/archdischild-2019-epa.122}

Introduction The presence of CYP24A1 mutations explains the increased sensitivity to vitamin $\mathrm{D}$ in patients with idiopathic infantile hypercalcemia and is a genetic risk factor for the development of symptomatic hypercalcemia that may be triggered by vitamin D prophylaxis in otherwise apparently healthy infants.

Case report We present a case of a 4-month old girl who was initially hospitalized for severe hypotonia, lethargy and failure to thrive. The patient's history revealed recurrent vomiting. On the clinical examination we also noted a high forehead, a high arched palate and short metacarpal bones. Blood tests showed hypercalcemia, low PTH and phosphate and high vitamin D levels. Renal ultrasound showed medullary nephrocalcinosis. Correlating the clinical examination with the blood work vitamin D intoxication, hyperparathyroidism and Jansen's metaphyseal dysplasia were considered and ruled out. Genetic testing was performed and a compound heterozygote state was identified (mutations p.E143del and p.R396W) confirming the diagnosis of idiopathic infantile hypercalcemia. Both mutations have been formerly identified as loss-of-function mutations in the vitamin D-24-hydroxylase gene. Rehydration and furosemide therapy was applied and resulted in the normalization of calcium values and clinical improvement. On the long term the patient followed a low calcium diet and vitamin D supplementation was discontinued.

Conclusion Clinical symptoms, such as failure to thrive, vomiting, increased thirst, anorexia, hypotonia should always bring in discussion the possibility of hypercalcemia in order to diagnose and treat idiopathic infantile hypercalcemia early and also to prevent long-term complications.

\section{GP57 ACUTE SUPERIOR MESENTERIC ARTERY SYNDROME - ASSOCIATION WITH GROWTH SPURTS}

${ }^{1}$ Mark Mahon, ${ }^{2,3}$ Shoana Quinn*. ${ }^{1}$ Trinity College Dublin, Dublin, Ireland; ${ }^{2}$ National Children's Hospital, Dublin, Ireland; 'Our Lady's Children's Hospital Crumlin, Dublin, Ireland

\subsection{6/archdischild-2019-epa.123}

Aim Superior Mesenteric Artery Syndrome is rare manifestation of duodenal obstruction arising due to external compression between the superior mesenteric artery and the aorta. The classic presentation involves postprandial abdominal pain and anorexia with weight loss. We aim to present the case of a 10-year-old female with an atypical presentation of acute Superior Mesenteric Artery Syndrome.

Method We report the comprehensive history highlighting pertinent positives and negatives, clinical examination and radiological investigations during our patient's inpatient admission.

Result Ten-year-old female, weighing $35.6 \mathrm{lbs}$ and height of $153.2 \mathrm{~cm}$ equating to a body mass index of 15.1 , with congenital adrenal hyperplasia and precocious puberty presented for elective admission for twenty-four-hour adrenal profile. On admission, patient reported worsening indigestion over past twelve months, epigastric pain and hiccups necessitating gastrointestinal consultation. Patient self-reported food to become 'stuck in the middle of her chest'. There was no associated nausea or vomiting or any recent weight loss reported. Symptoms failed to improve on a three-week course of proton pump inhibitors. A prior urea breath was negative. The patient was scheduled for barium swallow and follow through which demonstrated hold-up of the contrast in the second part of the duodenum, with the duodenal flexure in the correct position and no evidence of malrotation. After discussion as multidisciplinary conference the patient was re-admitted for elective oesophagogastroduodenoscopy. The results of which 
demonstrated a tighter transition from the second to third segment of the duodenum, with no mucosal abnormalities, consistent with extraluminal compression. Computed tomography with intravenous contrast was ordered which revealed reduced aortomesenteric angle and distance; findings which established the diagnosis of Superior Mesenteric Artery Syndrome.

Conclusion Most cases of Superior Mesenteric Artery Syndrome are presentations of chronic duodenal obstruction. Acute forms can occur in the face of trauma. Our patient is an interesting case, who continued to thrive with height and weight proportional, and was atraumatic. On review in outpatient facility recurrence of the epigastric pain was found to coincide with a recent growth spurt. A potential new association for exacerbation of asymptomatic chronic duodenal obstruction due to Superior Mesenteric Artery Syndrome.

\section{GP58 CASE REPORT OF HUNTER SYNDROME IN MONGOLIANS}

'Zolzaya Doljoo*, ${ }^{2}$ Sarantuya Jav, ${ }^{2}$ Purevdorj Ichinkhorloo, ${ }^{1}$ Batchimeg Batbaatar, 'Oyungerel Ganjuur, ${ }^{2}$ Munkhbat Batmunkh, ${ }^{1}$ Munkhtuvshin Namid, ${ }^{1}$ Munkhtuya Tumurkhuu. 'Institute of Medical Sciences, Ulaanbaatar, Mongolia; ${ }^{2}$ Mongolian National University of Medical Sciences, Ulaanbaatar, Mongolia

10.1136/archdischild-2019-epa.124

Background Mucopolysaccaridosis II (MPSII), Hunter syndrome, (OMIM\# 309900) is a metabolic genetic disorder caused by deficiency of iduronate-2-sulfatase (IDS) enzyme necessary for step-wise degradation of glycosaminoglycan (GAG) in a cell. Point mutations and small deletions of exon 9 of IDS gene account for 50\% of Hunter syndrome cases. Accumulation of GAG virtually in all cells causes MPS that is characterized by mental, growth progressive retardation, multiple system dysfunction and death usually occurs in first and second decade of life. MPS cases are diagnosed by only clinical symptoms in our country and detection of mutation, identification of defective enzymes, and carrier detection in the patient's family is still missing. Purpose of this study was to diagnose MPS cases by molecular genetic analysis and detect carriers in case if mutation detected.

Methods Iduronate-2 sulfatase enzyme activity was checked by tandem mass spectrometer. IDS gene mutation was analyzed in Hunter syndrome patients by direct sequencing. We used Multiplex Ligation-dependent Probe Amplification (MLPA) assay to detect $\times$ chromosome deletion in case if we could not amplify IDS gene.

Results Totally we investigated four patients with MPS, and familial members of a case 3, who had a mutation in IDS gene. Proband-E showed clinical symptoms of Hurler syndrome, but Hunter syndrome was suspected by genealogical study, also enzyme deficiency of iduronate 2 sulfatase was used to differentiate his diagnosis. We could not amplify exons of IDS gene in this patient sample, suggesting that he might have a deletion in $\times$ chromosome, and also we could not identify deletion covering Xq28 using SALSA MPLA probemix P095-A3 Aneuploidy. Proband-A is from inbred parents, in whom we suggest to have autosomal recessive mutant alleles. Proband-T showed clinical symptoms of Hunter syndrome and pedigree analysis and enzyme deficiency analysis confirmed it. Mutation screening was done in exon $2,3,8$ and 9 of IDS gene where most mutations reported in Hunter syndrome patients and a R468W mutation leading to a replacement of arginine 468 to tryptophan was detected in the patient. His mother carries mutant allele, as well as his three sisters. Proband I expresses clinical symptoms of Hunter syndrome and iduronate 2 sulfatase enzyme deficiency detected in blood samples which is confirmatory for Hunter syndrome.

Conclusion This is a first genetic analysis of MPS cases in Mongolia. We detected R468W mutation in the patient with severe phenotype of Hunter syndrome. We also confirmed four female carriers in this family.

\section{GP59 A RARE CAUSE OF 'MITOCHONDRIAL DISORDER': COCKAYNE SYNDROME}

${ }^{1}$ Arie Fisher* ${ }^{*}{ }^{1}$ Muhammad Asghar, ${ }^{2}$ Stephanie Ryan, ${ }^{3}$ Bryan Lynch, ${ }^{4}$ Andrew Green, ${ }^{1}$ Ina Knerr. 'National Centre for Inherited Metabolic Disorders, Temple Street Children's University Hospital, Dublin, Ireland; 'Department of Radiology, Temple Street Children's University Hospital, Dublin, Ireland; ${ }^{3}$ Department of Neurology, Temple Street Children's University Hospital, Dublin, Ireland; ${ }^{4}$ Department of Clinical Genetics, Temple Street Children's University Hospital, Dublin, Ireland

\subsection{6/archdischild-2019-epa.125}

A 16 month old toddler presented with global developmental delay, truncal hypotonia and microcephaly. Her background history was significant for bilateral developmental dysplasia of the hip, recurrent upper respiratory tract infections, conductive hearing loss and a scalp haemangioma. Development had been apparently normal up until 6 months of age.

Over the course of her follow-up she developed signs of cerebellar dysfunction including intention tremor and broad based ataxia, chronic feeding difficulties, pigmentary retinopathy and skin photosensitivity. MRI brain at age 2.5 years showed delayed myelination and cerebellar atrophy. A skeletal muscle biopsy revealed reduced activities for the respiratory chain complexes I, II and IV, in particular, in keeping with a mitochondrial condition. After extensive genetic investigations failed to identify the underlying aetiology, an exome sequencing study was carried out and revealed a mutation in the ERCC6 DNA repair gene, consistent with Cockayne syndrome (OMIM \# 133540). This is a rare autosomal recessive genetic condition which causes microcephaly, developmental delay and failure to thrive, along with sun sensitivity, tooth decay, bone abnormalities, hearing and vision loss and other symptoms. The patient has been managed with multidisciplinary care and symptomatic treatment, including skin protection, as well as nutritional and multivitamin supplements. She is wheelchair bound and non-verbal but otherwise medically well at age 7 yrs.

Many clinical signs seen in patients with Cockayne syndrome are also found in patients with a primary mitochondrial disease. Conversely, secondary dysfunctional mitochondria may also contribute to the phenotype of patients with Cockayne syndrome. Taken together, this case demonstrates the value of exome sequencing in diagnosing and differentiating rare disorders with diverse and overlapping phenotypes, including primary and secondary mitochondrial conditions, which are less likely to be diagnosed on clinical grounds and biochemical investigations alone. 